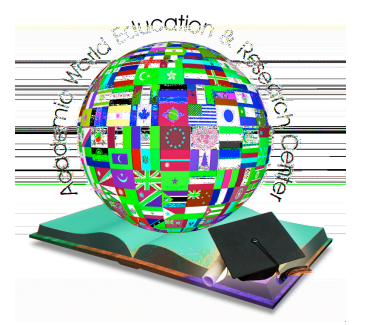

(U) Mlobal Journal of

www.awer-center/gjcs

\title{
Introducing a software to calculate the grade and ore deposit probability related problems
}

Seyyed Saeed Ghannadpour*

Ardeshir Hezarkhani,

Suggested Citation:

Global Journal of Computer Sciences 5

Abstract

Seyyed Saeed Ghannadpour

E-mail address: 


\section{Introduction}

\section{Theoretical principles}

2.1. Normal distribution

\subsubsection{Standardization of normal distribution}

$$
\begin{gathered}
\int_{-}\left(\mathrm{x}_{-} 1\right)^{\wedge}\left(\mathrm{x}_{-} 2\right) \equiv \mathrm{f}(\mathrm{x}) \mathrm{dx} \\
f(x)=1 /(\sigma \sqrt{2 \pi}) e^{\wedge}\left(-1 /\left(2((x-\mu) / \sigma)^{\wedge} 2\right)\right)
\end{gathered}
$$

$\mu$

$$
\mathrm{Z}=(\mathrm{x}-\mu) / \sigma
$$




$$
\mathrm{Z}=(\mathrm{x}-\mu) / \sigma
$$

$$
F(-z)=1-F(z)
$$
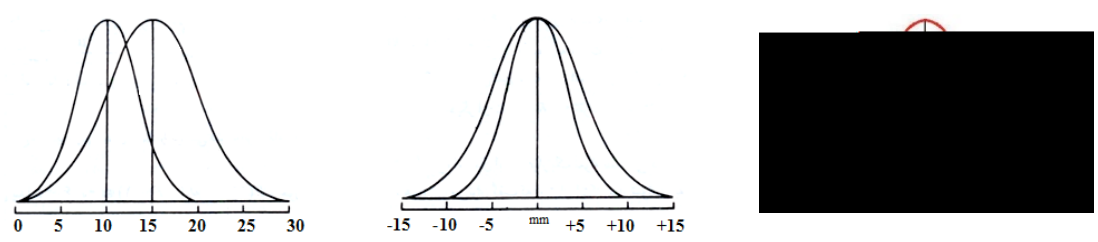

2. The tonnage of ore reserve and metal content of a certain cut-off grade 


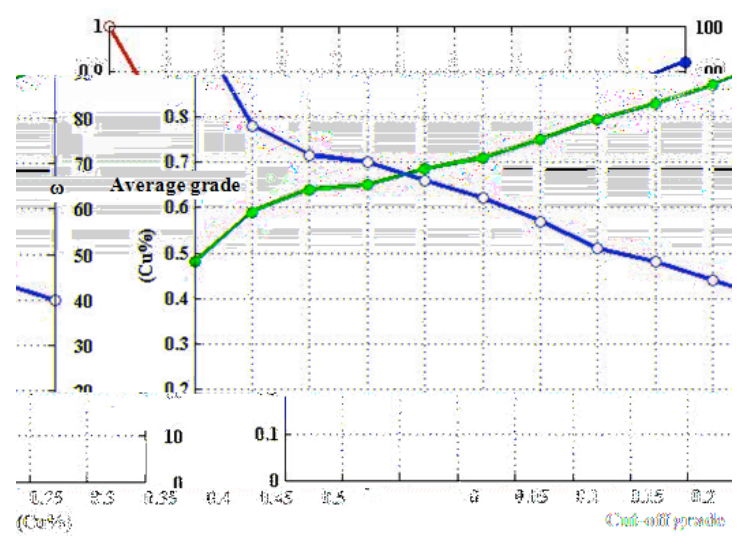

\begin{tabular}{|c|c|c|c|c|}
\hline $\begin{array}{c}\text { Cut-off grade greater } \\
\text { than mean grade }\end{array}$ & \multirow{2}{*}{$\begin{array}{c}\text { Altered cut-off } \\
\text { grade }\end{array}$} & \multicolumn{2}{|c|}{$\begin{array}{c}\text { Cut-off grade below } \\
\text { mean grade }\end{array}$} \\
\cline { 1 - 1 } $\begin{array}{c}\text { Ratio of ore } \\
\text { tonnage }\end{array}$ & $\omega$ & & $\omega$ & $\begin{array}{c}\text { Ratio of ore } \\
\text { tonnage }\end{array}$ \\
\hline 50 & & & & 50 \\
\hline 46.02 & 0.798 & 0 & 0.798 & 53.98 \\
\hline 42.07 & 0.863 & 0.1 & 0.735 & 57.93 \\
\hline 38.21 & 0.929 & 0.2 & 0.675 & 5.75 \\
\hline 34.45 & 0.998 & 0.3 & 0.617 & 6.79 \\
\hline 30.85 & 1.069 & 0.4 & 0.562 & 65.54 \\
\hline 27.43 & 1.142 & 0.5 & 0.509 & 69.15 \\
\hline 24.2 & 1.217 & 0.6 & 0.459 & 72.57 \\
\hline 21.19 & 1.375 & 0.7 & 0.411 & 75.8 \\
\hline 18.41 & 1.457 & 0.8 & 0.367 & 78.81 \\
\hline 15.87 & 1.542 & 0.9 & 0.326 & 81.59 \\
\hline 13.57 & 1.631 & 1 & 0.287 & 84.13 \\
\hline 11.51 & 1.724 & 1.1 & 0.251 & 86.43 \\
\hline 9.68 & 1.721 & 1.2 & 0.219 & 88.49 \\
\hline 8.08 & 1.923 & 1.3 & 0.189 & 90.32 \\
\hline 6.68 & 2.033 & 1.4 & 0.162 & 91.92 \\
\hline 5.48 & 2.147 & 1.5 & 0.138 & 93.32 \\
\hline 4.46 & 2.27 & 1.6 & 0.117 & 94.52 \\
\hline 3.59 & 2.403 & 1.7 & 0.098 & 95.54 \\
\hline 2.87 & 2.546 & 1.8 & 0.082 & 96.41 \\
\hline 2.28 & 2.701 & 1.9 & 0.067 & 97.13 \\
\hline 1.79 & 2.87 & 2 & 0.055 & 97.72 \\
\hline 1.39 & 3.055 & 2.1 & 0.045 & 98.21 \\
\hline & & 2.2 & 0.036 & 98.61 \\
\hline
\end{tabular}

\subsection{Normal distribution case}

$$
\begin{gathered}
F(z)=\int_{-} z^{\wedge} \infty \equiv e^{\wedge}\left(-t^{\wedge} 2 / 2\right) / \sqrt{ } 2 \pi d t \\
t_{-}\left(\left(x_{-} c\right)\right)=F\left(\left(x_{-} c-\mu\right) / \sigma\right) \\
x_{-}^{-}\left(\left(\mathrm{x}_{-} \mathrm{c}\right)\right)=\mu+\sigma \mathrm{e}^{\wedge}\left(-1 / 2\left(\left(\mathrm{x}_{-} \mathrm{c}-\mu\right) / \sigma\right)^{\wedge} 2\right) /\left(\sqrt{ } 2 \pi \mathrm{F}\left(\left(\mathrm{x}_{-} \mathrm{c}-\mu\right) / \sigma\right)\right) \\
\bar{x}_{-}^{-}\left(\left(\mathrm{x}_{-} \mathrm{c}\right)\right)=\mu+\sigma \omega \\
\omega=\boldsymbol{e}^{\wedge}\left(-1 / 2\left(\left(x_{-} c-\mu\right) / \sigma\right)^{\wedge} 2\right) /\left(\sqrt{ } 2 \pi F\left(\left(x_{-} c-\mu\right) / \sigma\right)\right)
\end{gathered}
$$

$\infty$

$z=\left(x_{-} c-\mu\right) / \sigma=(0.5-1.4) / \sqrt{ } 0.36=-1.5$

$F(-1.5)=1-F(1.5)=6.7$ 
Global Journal of Computer Sciences 5

$$
20 \times 0.9332=18.664 \approx 18.66
$$

$20 \times 0.9332=18.664 \approx 18.66$

$$
\omega=0 / 138
$$

$\overline{x_{-}}\left(\left(\mathrm{x}_{-} \mathrm{c}\right)\right)=\mu+\sigma \omega=1.4+0.138 \times \sqrt{.036}=1.483$

3. Probability, mean grade and tonnage of ore reserve calculation using MATLAB programming language

3.1. description of calculating the probability of normal distribution via MATLAB programming language

3.2. description of calculating the probability, tonnage of ore reserve and mean grade of normal distribution via MATLAB programming language

$\omega$

3.3. Main program 


\section{Conclusion}

\section{References}

Principles of Mineral Exploration

Exploration Data Analysis

Applied Statistics,

Geostatistical Ore Reserve Estimation

Songun Porphyry Copper Evaluation

Geostatistics, 